\title{
Mechanisms of recovery from aphasia: evidence from positron emission tomography studies
}

\author{
Elizabeth Warburton, Cathy J Price, Kate Swinburn, Richard J S Wise
}

\begin{abstract}
Objectives-Language functions comprise a distributed neural system, largely lateralised to the left cerebral hemisphere. Late recovery from aphasia after a focal lesion, other than by behavioural strategies, has been attributed to one of two changes at a systems level: a laterality shift, with mirror region cortex in the contralateral cortex assuming the function(s) of the damaged region; or a partial lesion effect, with recovery of perilesional tissue to support impaired language functions. Functional neuroimaging with PET allows direct observations of brain functions at systems level. This study used PET to compare regional brain activations in response to a word retrieval task in normal subjects and in aphasic patients who had shown at least some recovery and were able to attempt the task. Emphasis has been placed on single subject analysis of the results as there is no reason to assume that the mechanisms of recovery are necessarily uniform among aphasic patients.
\end{abstract}

Methods-Six right handed aphasic patients, each with a left cerebral hemispheric lesion (five strokes and one glioma), were studied. Criteria for inclusion were symptomatic or formal test evidence of at least some recovery and an ability to attempt word retrieval in response to heard word cues. Each patient underwent 12 PET scans using oxygen-15 labelled water $\left(\mathrm{H}_{2}{ }^{15} \mathrm{O}\right)$ as tracer to index regional cerebral blood flow (rCBF). The task, repeated six times, required the patient to think of verbs appropriate to different lists of heard noun cues. The six scans obtained during word retrieval were contrasted with six made while the subject was "at rest". The patients' individual results were compared with those of nine right handed normal volunteers undergoing the same activation study. The data were analysed using statistical parametric mapping (SPM96, Wellcome Department of Cognitive Neurology, London, UK).

Results-Perception of the noun cues would be expected to result in bilateral dorsolateral temporal cortical activations, but as the rate of presentation was only four per minute the auditory perceptual activations were not evident in all people. Anterior cingulate, medial premotor (supplementary speech area) and dorsolateral frontal activations were evident in all normal subjects and patients. There were limited right dorsolateral frontal activations in three of the six patients, but a similar pattern was also found in four of the nine normal subjects. In the left inferolateral temporal cortex, activation was found for the normal subjects and five of the six patients, including two of the three subjects with lesions involving the left temporal lobe. The only patient who showed subthreshold activation in the left inferolateral temporal activation had a very high error rate when performing the verb retrieval task.

Conclusions-The normal subjects showed a left lateralised inferolateral temporal activation, reflecting retrieval of words appropriate in meaning to the cue from the semantic system. Lateralisation of frontal activations to the left was only relative, with right prefrontal involvement in half of the normal subjects. Frontal activations are associated with parallel psychological processes involved in word retrieval, including task initiation, short term (working) memory for the cue and responses, and prearticulatory processes (even though no overt articulation was required). There was little evidence of a laterality shift of word retrieval functions to the right temporal lobe after a left hemispheric lesion. In particular, left inferolateral temporal activation was seen in all patients except one, and he proved to be very inefficient at the task. The results provide indirect evidence that even limited salvage of peri-infarct tissue with acute stroke treatments will have an important impact on the rehabilitation of cognitive functions.

(F Neurol Neurosurg Psychiatry 1999;66:155-161)

Keywords: stroke; aphasia; imaging; PET

Aphasia is common after left middle cerebral artery territory strokes and is perceived as a major disability by the patients and their relatives. ${ }^{2}$ Although recovery is usual, the degree is very variable. There are no reliable clinical predictors and the benefit of speech therapy, in its disparate forms, remains controversial. ${ }^{3}$ These uncertainties arise, in part, from ignorance about the brain mechanisms that lead to recovery. These mechanisms operate at many levels, from cell membranes up to distributed neural systems. Research at the lower reaches of the scale is dependent on animal studies, at a level that cannot, at present, be related to psychological processes-even simple cognitive functions cannot be described 
in terms of synapses and dendrites. However, at the systems level, modern functional neuroimaging techniques offer the potential to describe language and other higher mental functions in terms of regional human brain activity that may be used to disclose segregation (such as the location of the verbal semantic-word meaning - system) ${ }^{5}$; responsiveness (for example, whether regional brain activity responds incrementally or stepwise to a variable sensory input) ${ }^{6}$; or connectivity (when activity in one region can be shown to be influenced by activity in another). ${ }^{7}$ These techniques can be used to investigate both normal and abnormal brains, although to date the overwhelming majority of studies has been on normal subjects.

The changes in language systems that result in recovery from left hemispheric aphasic stroke have been a subject for speculation since the days of Broca. Although there are anecdotal clinical case reports implicating the right hemispere, ${ }^{8}$ the mechanisms are likely to be both complex and variable, depending on the deficit. ${ }^{9}$ Thus patients who have unilateral left hemispheric lesions can usually continue to discriminate words on the basis of their different sound structure; most reported cases of word sound deafness had bilateral dorsolateral temporal lesions. ${ }^{10}$ The inference is that the right dorsolateral temporal cortex can support, more or less, perception of the sound structure of words. ${ }^{11}$ However, this lesion based evidence does not distinguish between a pre-existing function which is shared by the two dorsal temporal regions or an acquired lateral shift of function after the stroke. By contrast, partial or complete loss of heard word comprehension accompanies left temporoparietal lesions, ${ }^{12}{ }^{13}$ which suggests that lexical semantic processes (that is, the interaction between the neural systems responsible for word form and word meaning) are predominantly left lateralised. Therefore, whether recovery depends on contralateral or ipsilateral (perilesional) hemispheric function may depend on whether the functional deficit is perceptual or cognitive.

Some early studies, using either single photon computed tomography (SPECT) or PET, have measured "resting" regional cerebral blood flow (rCBF) or metabolism and showed a diffuse increase in function of the contralateral hemisphere after a stroke and related this to recovery. ${ }^{14}{ }^{15}$ However, this is not the experience of others and many of these studies focused on very early recovery processes. ${ }^{16}$ Furthermore, aphasia recovery has been shown to correlate with residual, perilesional, left temporoparietal metabolism, ${ }^{17}$ which accords well with the finding that prognosis after a stroke can be predicted by the relation between flow and metabolism in and around the cerebral infarct. ${ }^{18}$

An activation PET study, which used verb retrieval as the behavioural task to disclose regional activity, implicated the right prefrontal cortex in recovery from Wernicke's aphasia, ${ }^{19}$ but this study did not disclose whether it was perilesional or contralateral activity that was involved in recovery from the left temporoparietal infarcts. The reason may be methodological - the field of view of the PET camera used was limited and ventral temporal regions were not studied. Alternatively, the null result in left temporoparietal regions may reflect activations distributed at individually different locations around infarcts of different sizes; under these circumstances there would be no result from the group analysis that was performed to look for a common regional periinfarct activation across the six patients.

The present study directly observed changes in brain systems during recovery from aphasia in six patients. The hypothesis was that the processes involved in word retrieval would not show a laterality shift of function to the right hemisphere in the presence of an acquired left hemispheric lesion. Verb retrieval was chosen as the activation task after a preliminary study in normal subjects. ${ }^{20}$ This task produces activations in all the cortical regions involved in language processing as determined from lesion data. ${ }^{21}$ It is, therefore, an activation task suited to investigate aphasic patients whose lesions are variously located in the left cerebral hemisphere.

\section{Patients and methods}

SUBJECTS

Six right handed patients with an equal sex distribution and mean age of 48 years (age range 28-69 years) were recruited from an aphasia clinic at the Charing Cross Hospital, London. Handedness was assessed using the

Table 1 Patient details and site of lesion (as defined on MRI) are shown in columns 2 and 3. In columns 4-8 examples of subtest scores are shown from each patient's formal language assessment nearest to their PET.

\begin{tabular}{|c|c|c|c|c|c|c|c|c|c|}
\hline \multirow[b]{2}{*}{ Patient } & \multirow[b]{2}{*}{ Age/sex } & \multirow[b]{2}{*}{ Site of lesion } & \multicolumn{5}{|c|}{ Comprehensive aphasia test (examples of subtest scores at optimal recovery) } & \multicolumn{2}{|c|}{ PET Performance } \\
\hline & & & $\begin{array}{l}\text { Ravens, } \\
\text { NR 5-9, } \\
\text { Max } 12\end{array}$ & $\begin{array}{l}\text { Auditory } \\
\text { comprehension, } \\
\text { NR 25-30, Max } \\
30\end{array}$ & $\begin{array}{l}\text { Naming, NR } \\
42-48, \text { Max } \\
48\end{array}$ & $\begin{array}{l}\text { Repetition, } \\
\text { NR } 30-32, \\
\text { Max } 32\end{array}$ & $\begin{array}{l}\text { Verbal fluency } \\
\text { (animals), NR } \\
11-24\end{array}$ & $\begin{array}{l}\text { Estimated } \\
\text { words/min }\end{array}$ & $\begin{array}{l}\text { Estimated } \\
\text { error rate }\end{array}$ \\
\hline 1 & $58 / \mathrm{F}$ & $\begin{array}{l}\text { L temporal lobe + inferior } \\
\text { parietal region }\end{array}$ & 12 & 28 & 32 & 25 & 19 & 12.3 & $<1 \%$ \\
\hline 2 & $28 / \mathrm{F}$ & L temporoparietal & 7 & 28 & 48 & 31 & 19 & 12.8 & $<1 \%$ \\
\hline 3 & $32 / \mathrm{F}$ & L temporoparietal (glioma) & 4 & 28 & 44 & 32 & 12 & 19.8 & $<1 \%$ \\
\hline 4 & $54 / \mathrm{M}$ & $\begin{array}{l}\text { L temporal lobe +inferior } \\
\text { parietal region }\end{array}$ & 2 & 28 & 32 & 28 & 10 & 16.7 & $48.4 \%$ \\
\hline 5 & $69 / \mathrm{M}$ & Subcortical ischaemia & 7 & 29 & 43 & 32 & 7 & 16.0 & $7.2 \%$ \\
\hline 6 & $64 / \mathrm{M}$ & L perisylvian region & 7 & 29 & 38 & 29 & 14 & 17.6 & $5 \%$ \\
\hline
\end{tabular}

The subtests are taken from the comprehensive aphasia test (CAT). The maximum possible scores (Max) are shown and also a normal range (NR), calculated from subtest scores on a cohort of normal subjects matched for age and sex. During PET a retrospective estimate of words generated was made in the wash out period between scans. The estimated rate of word retrieval/minute is shown in column 9 with an estimated error rate (given as a percentage of the total) in column 10 . Errors were taken as any words that were not verbs, eg nouns and adjectives but which were still semantically related to the target word. 


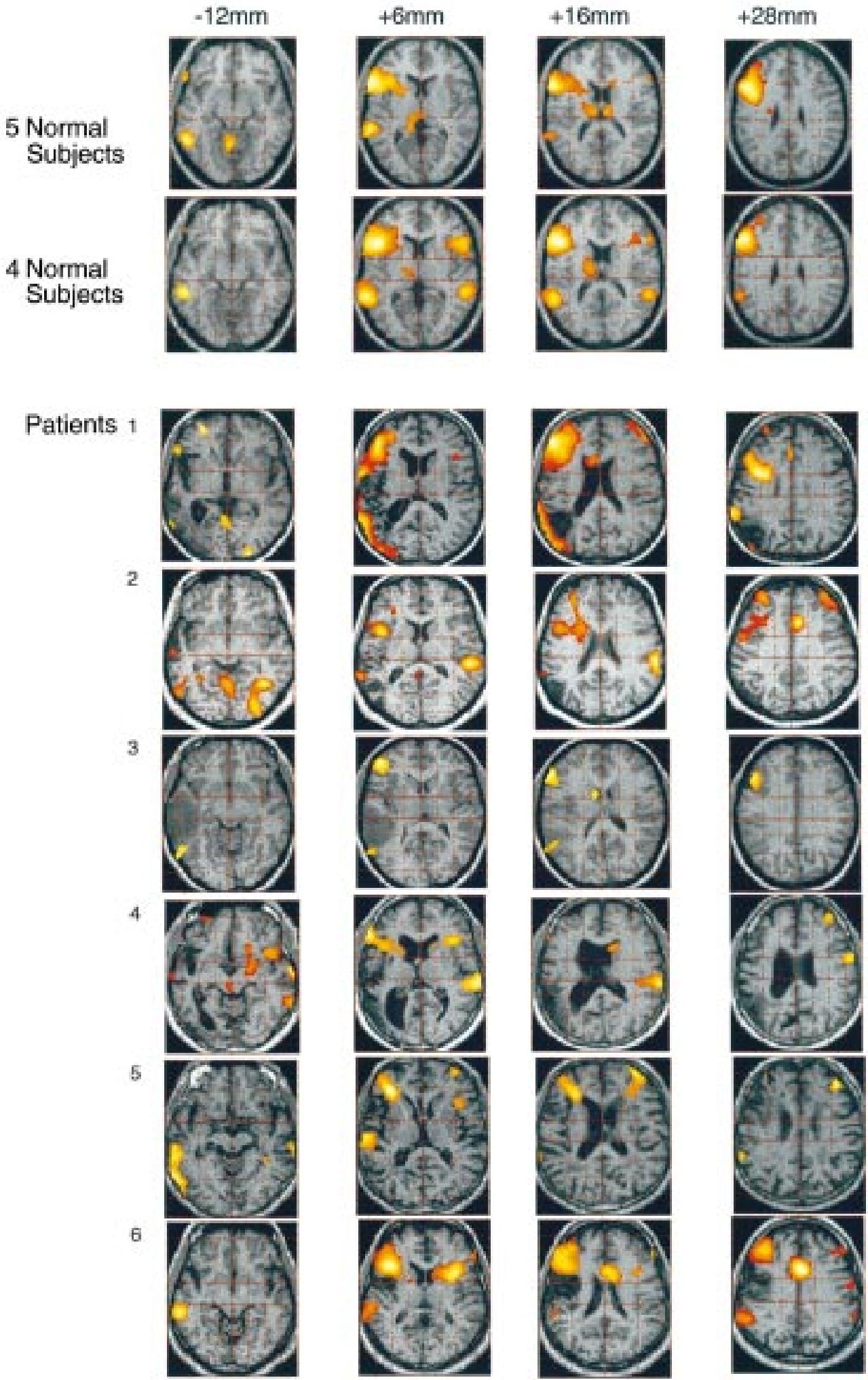

The areas activated when normal subjects and six patients retrieve verbs are illustrated on 4 transaxial slices of the brain $-12 \mathrm{~mm},+6 \mathrm{~mm},+16 \mathrm{~mm}$, and $+28 \mathrm{~mm}$ from the anterior-posterior commissural line. The left of the image represents the left hemisphere. The activation from the PET study has been coregistered with MRI images after normalisation of the MRI and PET data into Talaraich space. ${ }^{26}$ Highest regional cerebral blood flow $(r C B F)$ is coded in yellow and lowest in orange. The normal subjects are split into two groups depending on their individual activation profiles for this task. Each patient is referred to by a number.

Edinburgh inventory. ${ }^{22}$ All had experienced an acute onset of aphasia (and associated symptoms and signs, depending on the extent and distribution of the lesion) and all had speech therapy during recovery. They were selected from a larger cohort of patients because of test or symptomatic evidence of language recovery, sufficient to attempt word retrieval in response 
Table 2 Cortical activations in the four normal subjects with left and right lateral hemispheric activations with the $x, y$, and $z$ coordinates according to the atlas of Talairach and Tournoux, ${ }^{26}$ and $Z$ score for the peak voxel in each region

\begin{tabular}{lc}
\hline Left & Right \\
\hline Lateral frontal: & Inferior frontal gyrus and frontal operculum: \\
Prefrontal cortex & $54,22,8 ; Z=5.7$ \\
$-50,22,20 ; Z=7.3$ & \\
Premotor cortex and frontal operculum: & \\
$-50,-2,48 ; Z=6.3$ & Superior temporal gyrus: \\
Temporal: & $54,-44,10 ; Z=6.5$ \\
Superior temporal gyrus & \\
$-56,-48,18 ; Z=6.7$ & \\
Inferior temporal gyrus & \\
$-46,-42,-16 ; Z=3.9$ & \\
Parietal: & \\
Posterior, inferior lobe & Midline \\
$-40,-62,46 ; Z=3.8$ & Frontal: \\
& Anterior cingulate and medial premotor cortex \\
& $-4,18,48 ; Z=5.8$ \\
\hline
\end{tabular}

Table 3 Lateral cortical activations in the five normal subjects with only left lateral hemispheric activations with the $x, y$, and $z$ coordinates according to the atlas of Talairach and Tournoux, ${ }^{26}$ and $Z$ score for the peak voxel in each region

\begin{tabular}{|c|c|}
\hline \multicolumn{2}{|l|}{ Left } \\
\hline $\begin{array}{l}\text { Lateral frontal: } \\
\text { Prefrontal cortex } \\
\text {-44, 10, 36; } Z=5.7 \\
\text { Premotor cortex and fr } \\
\text {-50, 0, 46; } Z=5.3 \\
\text { Temporal: } \\
\text { Superior temporal gyru } \\
\text {-66, -38, } 4 ; Z=4.1 \\
\text { Inferior temporal gyrus } \\
\text {-56, -54, -16; } Z=4.0 \\
\text { Parietal } \\
\text { Posterior, inferior lobe } \\
\text {-38, -68, 52; } Z=3.7\end{array}$ & Midline \\
\hline & $\begin{array}{l}\text { Frontal: } \\
\text { and medial premotor cortex } \\
16,52 ; Z=5.9\end{array}$ \\
\hline
\end{tabular}

to heard word cues. They were assessed on a range of tests of language function, including auditory and written single word and sentence comprehension, picture naming, single word repetition, digit and sentence span, and category and initial letter fluency. Five patients had strokes (four infarcts and one haemorrhage) in the left middle cerebral artery territory. One (patient 3) presented with a stroke-like episode, initially followed by recovery for several months, but the lesion subsequently proved to be a left temporal glioma (there was no clinical or EEG evidence that the presentation was the temporary sequel to a focal or generalised seizure, although this possibility cannot be excluded). Of the six patients, two (patients 2 and 3) were shown on serial testing to reach ceiling scores on the test batteries within 6 months of the ictus. One (patient 5) had already symptomatically recovered from acute aphasia by the time of referral, and this was confirmed on formal testing. The other three (patients 1,4, and 6) showed only partial recovery on serial testing. All patients were scanned between 6 and 14 months after the onset of apahsia. The patients' performances on key items of the test battery at the time of their PET study are summarised in table 1, and the locations of their lesions on MRI are also summarised. The patients' results are contrasted with those obtained on nine right handed, normal, male subjects, aged 24-65 years.

\section{DATA ACOUISITION}

Each subject had 12 estimations of $\mathrm{rCBF}$ at 10 minute intervals, made with a CTI 953B PET camera. The conditions (verb retrieval and rest) were alternated (ABAB etc). Data acquisition was performed in 3D mode, with the lead septa between detector rings removed. For each scan $8 \mathrm{mCi}$ of $\mathrm{H}_{2}{ }^{15} \mathrm{O}$ was administered as a slow intravenous bolus, and the total counts/ voxel during the build up phase of radioactivity served as an estimate of $\mathrm{CBF}^{23}{ }^{24}$ The verb retrieval task began 15 seconds before the arrival of radiolabelled water in the brain, and continued during the critical measurement period of rapid build up of tracer in the brain and over the initial 20-30 seconds of tracer wash out. After measured attenuation correction, images were reconstructed by filtered back projection (Hanning filter, cut off frequency $0.5 \mathrm{~Hz}$ ).

During the verb generation condition, nouns were heard at a rate of four/minute and the subject was asked to think of, without vocalisation, as many verbs appropriate to the heard noun as she or he could in the interstimulus interval (for example, "apple"-_slice, eat, peel, wash"). No noun stimulus was repeated across the six scans during the verb retrieval task. The subjects were asked not to articulate their responses to avoid the coactivations associated with speech production and listening to one's own voice. During the "rest" condition the subject was asked to "empty your mind" and no stimuli were presented. A retrospective estimate of the number of verbs generated per list of stimuli was made in the interval immediately after completion of each of the six tasks. The normal subjects averaged 18.4 verbs/ minute (4.6/stimulus). The estimated verb retrieval rates for each patient are shown in table 1. Patient 5 misheard a few of the nouns. In addition, patient 5 and, in particular, patient 4 , made retrieval errors, producing nouns and adjectives as well as verbs (table 1).

IMAGE ANALYSIS

The data from each subject were realigned, stereotactically normalised, and smoothed using a $16 \times 16 \times 16 \mathrm{~mm}$ Gaussian filter. ${ }^{25}$ For each of the patients, all of whom had undergone MRI, the MRI was coregistered to his or her mean PET image and then stereotactically transformed to a standard MRI template in the Talaraich and Tournoux space. ${ }^{26}$ The same transformation matrix was subsequently applied to the PET images. The data were analysed using statistical parametric mapping (SPM96, Wellcome Department of Cognitive Neurology). ${ }^{27}$ The effect of differences in global CBF between scans was removed by an analysis of covariance (ANCOVA), with global counts as confounder. Linear contrasts between different conditions were used to create SPMs of the $t$ statistic (that were subsequently transformed into $\mathrm{Z}$ scores).

In this study, the six patients and the normal subjects were analysed in a single design matrix 
Table 4 Lateral cortical activations in the patients with the $x, y$, and $z$ coordinates according to the atlas of Talairach and Tournoux ${ }^{26}$ and $Z$ score for the peak voxel in each region

\begin{tabular}{|c|c|}
\hline Left & Right \\
\hline \multicolumn{2}{|l|}{1} \\
\hline \multicolumn{2}{|c|}{ Lateral frontal: } \\
\hline \multicolumn{2}{|c|}{ Prefrontal and premotor cortex and frontal cortex } \\
\hline$-54,38,16 ; Z=6.6$ & $54,42,16 ; Z=4.7$ \\
\hline \multicolumn{2}{|l|}{ Temporal } \\
\hline \multicolumn{2}{|l|}{ Superior temporal gyrus } \\
\hline \multicolumn{2}{|l|}{$-72,-34,12 ; Z=3.7$} \\
\hline \multicolumn{2}{|l|}{ Occipito-temporal junction } \\
\hline \multicolumn{2}{|l|}{$-66,-70,-4 ; Z=3.7$} \\
\hline 2 & \\
\hline \multicolumn{2}{|l|}{ Lateral frontal: } \\
\hline \multicolumn{2}{|c|}{ Prefrontal and premotor cortex and frontal cortex } \\
\hline$-44,8,8 ; Z=4.1$ & $40,54,28 ; Z=3.2$ \\
\hline \multicolumn{2}{|l|}{ Temporal: } \\
\hline \multicolumn{2}{|l|}{ Superior temporal gyrus } \\
\hline$-70,-44,4 ; Z=2.8$ & $66,-32,14 ; Z=5.2$ \\
\hline \multicolumn{2}{|l|}{ Occipitotemporal junction } \\
\hline$-58,-66,-16 ; Z=3.1$ & $42,-56,-14 ; Z=4.2$ \\
\hline \multicolumn{2}{|l|}{$\begin{array}{l}3 \\
\text { Erontal. }\end{array}$} \\
\hline \multirow{2}{*}{\multicolumn{2}{|c|}{$\begin{array}{l}\text { Frontal: } \\
-46,22,30 ; Z=4.4\end{array}$}} \\
\hline & \\
\hline \multicolumn{2}{|l|}{ Temporal: } \\
\hline \multirow{2}{*}{\multicolumn{2}{|c|}{$-72,-30,10 ; Z=3.3$}} \\
\hline & \\
\hline \multicolumn{2}{|l|}{ Inferolateral } \\
\hline \multirow{2}{*}{\multicolumn{2}{|c|}{$4^{-56,-68,-10 ; Z=3.7}$}} \\
\hline & \\
\hline \multicolumn{2}{|l|}{ Frontal: } \\
\hline$-62,30,8 ; Z=3.7$ & $62,28,10 ; Z=2.6$ \\
\hline \multicolumn{2}{|l|}{ Temporal: } \\
\hline \multicolumn{2}{|l|}{ Dorsolateral } \\
\hline$-68,-14,-6 ; Z=2.9$ & $70,-28,8 ; Z=6.4$ \\
\hline Inferolateral & \\
\hline$-54,-46,-16 ; Z=1.9$ & $68,-50,-20 ; Z=4.4$ \\
\hline 5 & \\
\hline Frontal: & \\
\hline$-58,16,40 ; Z=3.3$ & $44,46,30 ; Z=4.0$ \\
\hline Temporal: & \\
\hline Dorsolateral & \\
\hline$-66,-18,0 ; Z=5.2$ & $74,-26,-4 ; Z=4.4$ \\
\hline Inferolateral & \\
\hline$-68,-58,-12 ; Z=3.6$ & $62,-64,-20 ; Z=2.5$ \\
\hline 6 & \\
\hline Frontal & \\
\hline$-42,36,26 ; Z=5.7$ & $56,36,18 ; Z=4.3$ \\
\hline Temporal: & \\
\hline Dorsolateral & \\
\hline$-66,-24,0 ; Z=4.2$ & \\
\hline Inferolateral & \\
\hline$-64,-36,-10 ; Z=3.1$ & $74,-40,-6 ; Z=3.1$ \\
\hline
\end{tabular}

which allowed assessment of the contribution of individual subjects. The advantage of this approach is that it allows regions of common activation across subjects to be identified. Differences between patients and normal subjects were not assessed for the following reasons: (1) The "fixed effects models" that are almost universally used to analyse functional imaging data do not account for between subject variance and therefore tend to overestimate differences between two groups. (2) This study used a verbal fluency paradigm as a means for assessing word retrieval and the performance of the patients on this task could not be matched to the normal subjects. The analysis reported below is therefore concerned with the presence or absence of an activation in the patients in response to a task, not increased or decreased activation relative to normal subjects.

In the absence of an a priori hypotheses about where regional activations may occur, it is recommended that the threshold be set at $\mathrm{p}<0.05$, corrected for analyses of voxels across the whole volume of the brain (approximately, $\mathrm{p}<0.0001$, uncorrected, $\mathrm{Z}$ score $>4.65)$. However, based on the lesion data and previous functional neuroimaging results, summarised in the text, significant effects were expected to occur in the left temporal neocortex, the left inferior parietal lobe, and some medial and left lateralised regions, and for these defined regions the significance threshold was set at $\mathrm{p}<0.001$, uncorrected $(Z$ score $>3.1)$ for group results and $p<0.01$, uncorrected $(Z$ score $>2.3)$ for results from single subjects. For all other regions, significance was set at $Z$ score $>4.65$.

\section{Results}

NORMAL SUBJECTS

The results are presented in the top two rows of figure 1 and in tables 2 and 3. Dorsolateral temporal cortical activation (left and right) were attributed, on the basis of earlier functional neuroimaging evidence, to perception of the stimuli. ${ }^{28}{ }^{29}$ Other activations were due to the various parallel processes involved in word retrieval. Almost half of the subjects had bilateral activations in the dorsolateral frontal cortex, a result in common with another study using this same task in a larger population of subjects..$^{30}$ In the inferolateral temporal cortex, activation was lateralised to the left (although two of the subjects also activated the corresponding region in the right when the threshold was reduced to $\mathrm{p}<0.01$, uncorrected). Activation in the left has been directly attributed to the retrieval of words appropriate in meaning to each stimulus from semantic memory. ${ }^{20}$ The results of the control subjects are reported in two subgroups in tables 2 and 3. They were subdivided according to whether they showed predominantly unilateral or bilateral acivations. This was done by analysing each individual subject and searching for activations (particularly in the right temporal and inferior frontal regions) which survivied an analysis using a peak threshold of $\mathrm{p}<0.01$ and an extent threshold of 50 voxels.

PATIENTS

The results are presented in the bottom six rows of the figure and in table 4 .

All six patients engaged the left dorsolateral frontal cortex to perform the verb generation task and three (patients 4, 5, and 6) also showed variable degrees of activation in the right dorsolateral frontal cortex. In the left temporal structures all but one patient (patient 4) showed significant activation. Figure 1, column 1 shows that there was consistent activation of the left posterior inferolateral temporal cortex in all patients except patient 4 , and figure 1, columns 2 and 3 demonstrate variable degrees of peri-infarct activation in the left dorsolateral temporal cortex of the patients. On the right, three patients (patients 2, 3 , and 4) showed involvement of the right dorsolateral temporal cortex (this corresponded to a similar activation in four of the normal subjects) and four patients (patients 2, 4, 5, and 6 ) showed significant activation of the right inferolateral temporal cortex, (a region activated by two of the nine normal subjects).

There was uniform activation of the anterior cingulate in both normal subjects and patients. Of the subcortical nuclei, the left thalamus was activated in eight out of the nine controls and 
three out of the six patients. Significant activation of the right putamen occurred in only one of the patients (patient 4) and none of the controls.

\section{Discussion}

The activations associated with cued word retrieval were indistinguishable from the normal controls, except that in the presence of a lesion the activations were perilesional. The activation that was exclusively left lateralised in the normal controls - in the inferolateral temporal cortex-was also seen in the six patients, with the one exception (patient 4), and he proved to be very inefficient at retrieving verbs to the cues. The variable degree of lateralisation of activations in regions other than the inferolateral temporal cortex shows the importance of single subject analysis for patient studies. Thus, all nine of the normal subjects must have activated the bilateral dorsolateral temporal cortex when perceiving the stimuli, ${ }^{6}$ but these effects were only apparent in four of them-variable individual reponsiveness in these regions to the slow rate of presentation of the stimuli resulted in a reduced effect in the other five. This pattern was also evident in the patients - for example, there was no apparent activation of the right dorsolateral temporal cortex in patient 1 but it was readily evident in patient 2. The detection of a change in net regional synaptic activity by measuring a change in regional perfusion will vary, depending on both physiological and technical factors, especially when the response is small-there is a linear change in activity in the dorsolateral temporal cortex in response to hearing single words at increasing rates, and so listening to four stimuli/minute will have produced a response that was well below maximum in all, normal subjects and patients alike. It proved to be below the threshold of detection in more than half of them.

The subjects were encouraged to think of as many verbs as they could for each stimulus, thereby maximising the activations associated with silent word retrieval (output) relative to perceiving the stimuli (input). The right sided dorsolateral frontal activation present in four of the normal subjects was restricted to the frontal operculum and inferior frontal gyrus, whereas the left sided activation was more extensive, including the frontal operculum, and premotor and dorsolateral prefrontal cortex. The location of the right frontal activation is best interpreted in terms of prearticulatory processes, short of articulation. Thus, Mohr et al have shown in stroke patients that a lesion close to or within the left frontal operculum results in a loss of articulation, usually only temporary ${ }^{31}$. Rarer cases with bilateral lesions of the frontal operculum have severe and persistent impairment of speech production without aphasia. ${ }^{32}$ Those with loss of self generated word retrieval (verbal fluency) without impairment of speech production have left prefrontal lesions. ${ }^{33}$ Therefore, the extensive left frontal activation in the normal subjects can be interpreted in terms of the "strategic" processes involved in searching for and retriev- ing appropriate exemplars to match a stimulus, with the more ventral extent of this activation involved in processes involved in preparation for the articulation of the exemplars. In some subjects, these prearticulatory processes were disclosed as bilateral opercular/inferior frontal activations.

The pattern of frontal activations in the patients did not fall outside the variable pattern found in the normal controls, with one exception (patient 4). Although he showed bilateral opercular activations during the verb retrieval task, there was no evidence of left dorsolateral prefrontal activation. This is by contrast with patient 1 , who showed very extensive left lateralised frontal activation. Both had left posterior lesions, with similar performance scores on the aphasia tests, but they differed in their performance on verb retrieval-although their rates of retrieving exemplars were equivalent, patient 4 produced a high proportion of inappropriate exemplars. As the posterior and anterior regions are interdependent parts of a distributed system for self generated word retrieval, incorporating the many processes involved in the task, it is to be expected that success at fluency in patients with left temporoparietal lesions will be as dependent on left prefrontal activation as posterior, perilesional activity. Neither patients 4 or 1 had a frontal lesion evident on MRI, but in these two patients success at word retrieval can be related as much to left dorsolateral prefrontal activation as to the extent of perilesional left inferolateral temporal activation. These two cases suggest that a larger functional neuroimaging project of the same design on patients with left temporal infarcts should investigate the contribution of both left frontal and left temporal function to recovery of verbal fluency. It is known that success at verbal fluency is dependent on many factors, including age, education, and IQ, and in this context it is of interest that patient 1 was very much better at Raven's progressive matrices (a test of nonverbal reasoning that correlates reasonably closely with premorbid general intellectual abilities) than patient 4 . The hypothesis that prefrontal functions may have an influence on recovery after a posterior aphasic infarct would seem worth investigating further with functional neuroimaging, even on the basis of this anecdote-particularly as behavioural training or pharmacological manipulation might be used to manipulate prefrontal functions in the intact frontal lobe to maximise left posterior perilesional function in tasks such as word retrieval.

There was consistency of anterior cingulate activation across both normal subjects and patients. Activation of this region during a verb generation task has been attributed to the attentional components of the $\operatorname{task}^{20} 29$ and therefore activation of this region was expected-especially as the patients found the task much harder to perform than the normal subjects.

Evidence from both lesion data and functional neuroimaging suggests that activations in the left inferolateral temporal cortex repre- 
sent word retrieval from the mental representations of the meanings of words (the verbal semantic system). ${ }^{203435}$ The results from the patients in this study suggest that recovery of accurate self generated word retrieval after a left hemispheric lesion is dependent on perilesional tissue and not a laterality shift of function. This corresponds to both data on the effect of the extent of lesions on semantic function after stroke and from metabolic studies with PET that equate stroke recovery with "penumbral" tissue function. ${ }^{17}{ }^{18} 36 \mathrm{~A}$ recent longitudinal study in recovering aphasic patients showed that a favourable outcome was related to the reactivation of left hemispheric speech areas surrounding the area of infarction. ${ }^{37}$ The importance of peri-infarct regions in recovery has also been suggested in a single case study using PET activation to study the recovery from auditory agnosia. ${ }^{38}$ The many potential therapies directed at protecting perilesional tissue may only salvage a small volume of tissue when used in a clinical situation, ${ }^{39}$ especially when there may be delays in initiating therapy, but this study emphasises the importance for functional recovery of the peri-infarct region.

We thank the patients, their relatives, and the normal volunteers who took part in these experiments. We also thank the radiographers and members of the PET methods section at the MRC Cyclotron Unit. EAW was an MRC Training Fellow, CJP and RJSW are funded by the Wellcome Trust and KS was funded by North West Thames Regional Health Authority.

1 Wade DT, Wood VA, Hewer RL. Recovery after stroke - the first three months. I Neurol Neurosurg Psychiatry 1985;48:7-13.

2 Pedersen PM, Jorgensen HS, Nakayama H, et al. Aphasia in acute stroke: incidence, determinants and recovery. Ann Neurol 1995;38:659-66.

3 Kertesz A, McCabe P. Recovery patterns and prognosis in aphasia. Brain 1977;100:1-18.

4 Enderby P, Emerson J. Speech and language therapy: does it work? BMF 1996;312:1655-8.

5 Vandenberghe R, Price C, Wise R, et al. Functional anatomy of a common semantic system for words and pictures. of a common semantic

6 Price C, Wise R, Ramsay S, et al. Regional response differences within the human auditory cortex when listening to words. Neurosci Lett 1992;146:179-82.

7 Friston KJ. Functional and effective connectivity in neuroimaging: a synthesis. Human Brain Mapping 1994;2 56-78.

8 Basso A, Gardelli M, Pia Grassi M, et al. The role of the right hemisphere in the recovery from aphasia: two case studies. Cortex 1989;25:555-66.

9 Benson DR, Ardila A, eds. Recovery from aphasia. In: Aphasia a clinical perspective Oxford: Oxford University Press 1996:343-4.

10 Buchman AS, Garron DC, Trost-Cardamone JE, et al. Word deafness: one hundred years later. 7 Neurol Neurosurg deafness: one hundred years

11 Praamstra P, Hagoort P, Maassen B, et al. Word deafness and auditory cortical function: a case history and and auditory cortical function: a

12 Alexander MP, Hiltrunner B, Fischer RS. Distributed anatomy of transcortical sensory aphasia. Arch Neurol 1989;46: 885-92.
13 Hart J Jr, Gordon B. Delineation of single-word semantic comprehension deficits in aphasia, with anatomical correlation. Ann Neurol 1990;27:226-31.

14 Knopman DS, Rubens AB, Selnes UA, et al. Mechanisms of recovery from aphasia: evidence from serial xenon-133 cerebral blood flow studies. Ann Neurol 1984;15:530-5.

15 Cappa SF, Perani D, Grassi F, et al. A PET follow up study of recovery after stroke in acute aphasics. Brain Lang 1997; 56:55-67.

16 Iglesias S, Marchal G, Rioux P, et al. Do changes in oxygen metabolism in the unaffected cerebral hemisphere underlie early neurological recovery after stroke? A positron emission tomography study. Stroke 1996;27:1192-9.

17 Fink GR, Herholtz K, Pietrzyk U, et al. Peri-infarct perfusion in human ischemia: its relation to tissue metabolism, morphology, and clinical outcome. fournal of Stroke and Cerebrovascular Disorders 1993;3:123-31.

18 Furlan M, Marchal G, Viader F, et al. Spontaneous neurological recovery after stroke and the fate of the ischemic penumbra. Ann Neurol 1996;40:216-26.

19 Weiller C, Insensee C, Rijntjes M, et al. Recovery from Wernicke's aphasia: a positron emission tomography study. Ann Neurol 1995;37:723-32.

20 Warburton EA, Wise RJS, Price CJ, et al. Studies with positron emission tomography of noun and verb retrieval in normal subjects. Brain 1996;119:159-80.

21 Mesulam MM. Large-scale neurocognitive networks and distributed processing for attention, language, and distributed processing for attention,
memory. Ann Neurol 1990;28:597-613.

22 Oldfield RC. The assessment and analysis of handedness: the Edinburgh inventory. Neuropsychologia 1971;9:97-113.

23 Fox PT, Mintun MA. Non-invasive functional brain mapping by change-distribution analysis of averaged PET images of $\mathrm{H}_{2}{ }^{15} \mathrm{O}$. F $\mathrm{Nucl}$ Med 1989;30:141-9.

24 Mazziotta JC, Huang S-C, Phelps ME, et al. A non-invasive positron computed tomography technique using oxygen-15 abelled water for the evaluation of neurobehavioural task batteries. F Cereb Blood Flow Metab 1985;5:70-8.

25 Friston KJ, Ashburner J, Frith CD, et al. Spatial realignment and normalization of images. Human Brain Mapping 1995; $2: 165-89$

26 Talaraich P, Tournoux J. A stereotactic coplanar atlas of the human brain. Stuttgart: Thieme, 1988.

27 Friston KJ, Holmes AP, Worsley KJ, et al. Statistical parametric maps in functional imaging: a general linear approach. Human Brain Mapping 1995;2:189-210.

28 Petersen SE, Fox PT, Posner MI, et al. Positron emission tomographic studies of the cortical anatomy of single-word processing. Nature 1988;331:585-9.

29 Wise R, Chollet F, Hadar U, et al. Distribution of cortical neural networks involved in word comprehension and word retrieval. Brain 1991;114:1803-17.

30 Frackowiak RSJ. An European activation study of verbal fluency: results from a multicentre PET experiment. EU Concerted Action on Functional Imaging. 7 Cereb Blood Flow Metab 1995;15(suppl 1):S52.

31 Mohr JP, Pessin MS, Finkelstein S, et al. Broca aphasia: pathologic and clinical. Neurology 1978;28:311-24.

32 Weller M. Anterior opercular cortex lesions cause dissociated lower cranial nerve palsies and anarthria but no aphasia: Foix-Chavany-Marie syndrome and automatic voluntary dissociation revisited. F Neurol 1993;240:199208.

33 McCarthy RA, Warrington EK. Cognitive neuropsychology-a clinical introduction. San Diego, CA: Academic Press, 1990.

34 Martin A, Haxby J, Lalonde F, et al. Discrete cortical regions associated with knowledge of colour and knowledge of action. Science 1995;270:102-5.

35 Damasio H, Grabowski TJ, Tranel D, et al. A neural basis for lexical retrieval. Nature 1996;380:499-505.

36 Naeser MA, Helm-Estabrooks N, Haas G, et al. Relationship between lesion extent in Wernicke's area on computed tomographic scan and predicting recovery of comprehension in Wernicke's aphasia. Arch Neurol 1987;44:73-82.

37 Heiss W-D, Karber H, Weber-Luxenburger G, et al. Speechinduced cerebral metabolic activation reflects recovery from aphasia $\mathcal{F}$ Neurol Sci 1997;145:213-17.

38 Engelein A, Silbersweig D, Stern E, et al. The functional anatomy of recovery from auditory agnosia. Brain 1995; 118:1395-409.

39 Koroshetz WJ, Moskowitz MA. Emerging treatments for strokes in humans. Trends Pharmacol Sci 1996;17:227-33. 